\title{
On the existence of the mild solution for semilinear nonlocal fractional Cauchy problem
}

\author{
Samer Al Ghour ${ }^{a, *}$, Ahmad Al Omari ${ }^{b}$ \\ ${ }^{a}$ Department of Mathematics and Statistics, Jordan University of Science and Technology, Irbid 22110, Jordan. \\ ${ }^{b}$ Department of Mathematics, Faculty of Science, Al al-Bayt University, P. O. Box 7, Mafraq 61710, Jordan.
}

Communicated by D. Turkoglu

\begin{abstract} integer order:

$$
\begin{aligned}
& u^{\alpha}(t)=A u(t)+f\left(t, u_{t}\right) \text { where } \alpha \in(0,1] \text { and } t \in(0, a] ; \\
& u\left(\tau_{k}+0\right)=Q_{k} u\left(\tau_{k}\right) \equiv u\left(\tau_{k}\right)+I_{k} u\left(\tau_{k}\right), k=1,2, \ldots, K ; \\
& u(t)+\left(g\left(u_{t_{1}}, \ldots, u_{t_{p}}\right)\right)(t)=\phi(t), \text { where } t \in[-r, 0] .
\end{aligned}
$$
\end{abstract}

We consider the nonlocal Cauchy problem for the semilinear functional differential equation with non-

Under suitable conditions we prove the existence and uniqueness of a mild solution to the equation. (C)2016 All rights reserved.

Keywords: Cauchy problem, mild solution, impulsive functional, fractional differential equation, fixed point.

2010 MSC: 34A37, 34G20, 34K30, 34K99.

\section{Introduction and Preliminaries}

Fractional differential equations have gained importance and popularity during the past three decades or so, mainly due to the demonstrated applications in numerous seemingly diverse fields of science and engineering. For example, the nonlinear oscillation of earthquake can be modeled with fractional derivatives [4], and the fluid-dynamic traffic model with fractional derivatives [6] can eliminate the deficiency arising

\footnotetext{
${ }^{*}$ Corresponding author

Email addresses: algore@just.edu.jo (Samer Al Ghour), omarimutah1@yahoo.com (Ahmad Al Omari)
} 
from the assumption of continuum traffic flow. Based on experimental data fractional partial differential equations for seepage flow in porous media are suggested in [5], and differential equations with fractional order have recently proved to be valuable tools to the modeling of many physical phenomena [8], 9]. In this paper, we study the existence and uniqueness of a mild solution of a nonlocal Cauchy problem of noninteger order for impulsive functional differential evolution equation. Such problems arise in some physical applications as a natural generalization of the classical initial value problems. The results for semilinear functional differential evolution nonlocal problem [2] are extended for the case of impulse effect. We consider the nonlocal Cauchy problem of non-integer order in the form.

$$
\begin{aligned}
& u^{\alpha}(t)=A u(t)+f\left(t, u_{t}\right), t \in(0, a], t \neq \tau_{k}, \\
& u\left(\tau_{k}+0\right)=Q_{k} u\left(\tau_{k}\right) \equiv u\left(\tau_{k}\right)+I_{k} u\left(\tau_{k}\right), k=1,2, \ldots, K, \\
& u(t)+\left(g\left(u_{t_{1}}, \ldots, u_{t_{p}}\right)\right)(t)=\phi(t), t \in[-r, 0],
\end{aligned}
$$

where $0<t_{1}<\ldots<t_{p} \leq a(p \in \mathbf{N}) ; A$ and $I_{k}(k=1,2, \ldots, K)$ are linear operators acting in a Banach space $E ; f, g$, and $\phi$ are given functions satisfying some assumptions, and $u_{t}(s):=u(t+s)$ for $t \in[0, a]$, $s \in[-r, 0], I_{k} u\left(\tau_{k}\right)=u\left(\tau_{k}+0\right)-u\left(\tau_{k}-0\right)$ and the impulsive moments $\tau_{k}$ are such that $0<\tau_{1}<\ldots<\tau_{k}<a$ $(k \in \mathbf{N})$.

As usual, in the theory of impulsive differential equations [7], [10] at the points of discontinuity $\tau_{i}$ of the solution $t \rightarrow u(t)$ we assume that $u\left(\tau_{i}\right) \equiv u\left(\tau_{i}-0\right)$. In general, it is clear that the derivatives $u^{\alpha}\left(\tau_{i}\right)$ do not exist. On the other hand, according to the first equality (1.1), the limits $u^{\alpha}\left(\tau_{i} \pm 0\right)$ exist. According to the above convention, we assume $u^{\alpha}\left(\tau_{i}\right) \equiv u^{\alpha}\left(\tau_{i}-0\right)$.

Throughout this paper, we assume that $E$ is a Banach space with norm $\|\cdot\|, A$ is the infinitesimal generator of a $C_{0}$ semigroup $\{T(t)\}_{t \geq 0}$ on $E, D(A)$ is the domain of $A$ and $M=\sup _{t \in[0, a]}\left\{\|T(t)\|_{B L(E, E)}\right\}$.

In what follows we make the following assumptions:

(H1) For $f:[0, a] \times C([-r, 0], E) \rightarrow E, t \in[0, a]$ and $w \in C([-r, a], E), f\left(., w_{t}\right) \in C([0, a], E)$;

(H2) for $f:[0, a] \times C([-r, 0], E) \rightarrow E$, there exist constants $L_{1}, L_{2}>0$ such that $\left\|f\left(t, w_{t}\right)-f\left(t, \widetilde{w_{t}}\right)\right\|_{E} \leq$ $L_{1}\|w-\widetilde{w}\|_{C([-r, t], E)}$ and $\left\|I_{k} v\right\|_{E} \leq L_{2}\|v\|_{E}$ for all $w, \widetilde{w} \in C([-r, a], E), t \in[0, a], v \in E$ and $k=$ $1,2, \ldots, K$

(H3) for $g:[C([-r, 0], E)]^{p} \rightarrow C([-r, 0], E)$ there exists a constant $K^{*}>0$ such that

$\left\|\left(g\left(w_{t_{1}}, \ldots, w_{t_{p}}\right)\right)(s)-\left(g\left(\widetilde{w}_{t_{1}}, \ldots, \widetilde{w}_{t_{p}}\right)\right)(s)\right\| \leq K^{*}\|w-\widetilde{w}\|_{C([-r, a], E)}$ for all $w, \widetilde{w} \in C([-r, a], E), s \in$ $[-r, 0]$

(H4) $\phi \in C([-r, 0], E)$.

The following three definitions will be used in the sequel, for more details see [1].

Definition 1.1. The Riemann-Liouville fractional integral operator of order $\alpha \geq 0$, of a function $f \in C_{\mu}$, $\mu \geq-1$ is defined as

$$
\begin{aligned}
I^{\alpha} f(x) & =\frac{1}{\Gamma(\alpha)} \int_{0}^{x}(x-t)^{\alpha-1} f(x) d t, \alpha \geq 0, x>0, \\
I^{0} f(x) & =f(x) .
\end{aligned}
$$

The Riemann-Liouville derivative has certain disadvantages when trying to model real-world phenomena with fractional differential equations. Therefore, we shell introduce a modified fractional differential operator $D_{*}^{\alpha}$ proposed by M. Caputo in his work on theory of viscoelasticity [3].

Definition 1.2. The fractional derivative of $f(x)$ in the Caputo sense is defined as

$$
D_{x}^{\alpha} f(x)=I^{m-\alpha} D^{m} f(x)=\frac{1}{\Gamma(m-\alpha)} \int_{0}^{x}(x-t)^{m-\alpha-1} f^{m}(t) d t \text { for } m-1 \leq m, m \in \mathbf{N}, x>0 \text { and } f \in C_{-1}^{m} .
$$


Definition 1.3. A function $u \in C([-r, a], E)$ satisfying the following conditions:

1.

$$
\begin{aligned}
u(t)= & T(t) \phi(0)-T(t)\left[\left(g\left(u_{t_{1}}, \ldots, u_{t_{p}}\right)\right)(0)\right]+\frac{1}{\Gamma(\alpha)} \int_{0}^{t}(t-s)^{\alpha-1} T(t-s) f\left(s, u_{s}\right) d s \\
& +\sum_{0<\tau_{k}<t} T\left(t-\tau_{k}\right) I_{k} u\left(\tau_{k}\right) \text { for } t \in[0, a],
\end{aligned}
$$

2.

$$
u(t)+\left(g\left(u_{t_{1}}, \ldots, u_{t_{p}}\right)\right)(t)=\phi(t) \text { for } t \in[-r, 0),
$$

will be called a mild solution of a nonlocal Cauchy problem.

The following lemma will be used in the sequel. Its proof follows easily and we leave it to the reader.

Lemma 1.4. The nonlocal Cauchy problem (1.1) is equivalent to the nonlinear integral equation

$$
\begin{aligned}
u(t)= & T(t) \phi(0)-T(t)\left[\left(g\left(u_{t_{1}}, \ldots, u_{t_{p}}\right)\right)(0)\right]+\frac{1}{\Gamma(\alpha)} \int_{0}^{t}(t-s)^{\alpha-1} A u_{s} d s \\
& +\frac{1}{\Gamma(\alpha)} \int_{0}^{t}(t-s)^{\alpha-1} f\left(s, u_{s}\right) d s \text { for } t \in[0, a] \text { and } \\
u(t)+ & \left(g\left(u_{t_{1}}, \ldots, u_{t_{p}}\right)\right)(t)=\phi(t) \text { for } t \in[-r, 0) .
\end{aligned}
$$

In other words, every solution of the integral equation (1.3) is also solution of the our original nonlocal Cauchy problem (1.1) and vice versa.

\section{Results}

Theorem 2.1. Assume that the functions $f, g$ and $\phi$ satisfy assumptions (H1)-(H4). Additionally, suppose that: $M\left(K^{*}+t^{\alpha} L_{1}+p L_{2}\right)<1$. Then the nonlocal Cauchy problem (1.1) has a unique mild solution.

Proof. The mild solution of (1.1) with nonlocal condition can be written in the form

$u(t, \phi)=(F u)(t)$, where $(F w)(t)= \begin{cases}\phi(t)-\left(g\left(w_{t_{1}}, \ldots, w_{t_{p}}\right)\right)(t) & \text { if } t \in[-r, 0) ; \\ T(t) \phi(0)-T(t)\left[\left(g\left(w_{t_{1}}, \ldots, w_{t_{p}}\right)\right)(0)\right] & \\ +\frac{1}{\Gamma(\alpha)} \int_{0}^{t}(t-s)^{\alpha-1} T(t-s) f\left(s, w_{s}\right) d s, & \text { if } t \in[0, a], \\ +\sum_{0<\tau_{k}<t} T\left(t-\tau_{k}\right) I_{k} w\left(\tau_{k}\right) & \end{cases}$

where $w \in C([-r, a], E)$ and $F: C([-r, a], E) \rightarrow C([-r, a], E)$. We show that $F$ is a contraction mapping on $C([-r, a], E)$ :

Let $w, \widetilde{w} \in C([-r, a], E)$. For every $t \in[-r, 0)$,

$$
(F w)(t)-(F \widetilde{w})(t)=\left(g\left(\widetilde{w}_{t_{1}}, \ldots, \widetilde{w}_{t_{p}}\right)\right)(t)-\left(g\left(w_{t_{1}}, \ldots, w_{t_{p}}\right)\right)(t)
$$

and by assumption $(\mathrm{H} 3)$ we have $\|(F w)(t)-(F \widetilde{w})(t)\| \leq K^{*}\|w-\widetilde{w}\|_{C([-r, a], E)}$. 
Also, for every $t \in\left[t_{0}, a\right]$ we have

$$
\begin{aligned}
(F w)(t)-(F \widetilde{w})(t)= & T(t)\left[\left(g\left(\widetilde{w}_{t_{1}}, \ldots, \widetilde{w}_{t_{p}}\right)\right)(0)-\left(g\left(w_{t_{1}}, \ldots, w_{t_{p}}\right)\right)(0)\right] \\
& +\frac{1}{\Gamma(\alpha)} \int_{0}^{t}(t-s)^{\alpha-1} T(t-s)\left[f\left(s, w_{s}\right)-f\left(s, \widetilde{w}_{s}\right)\right] d s \\
& +\sum_{0<\tau_{k}<t} T\left(t-\tau_{k}\right)\left[I_{k} w\left(\tau_{k}\right)-I_{k} \widetilde{w}\left(\tau_{k}\right)\right]
\end{aligned}
$$

Therefore, using assumptions (H2) and (H3)we have

$$
\begin{aligned}
\|(F w)(t)-(F \widetilde{w})(t)\| \leq & \|T(t)\| \|\left[\left(g\left(\widetilde{w}_{t_{1}}, \ldots, \widetilde{w}_{t_{p}}\right)\right)(0)-\left(g\left(w_{t_{1}}, \ldots, w_{t_{p}}\right)\right)(0) \|\right] \\
& +\frac{1}{\Gamma(\alpha)} \int_{0}^{t}\left\|(t-s)^{\alpha-1} T(t-s)\right\|\left\|f\left(s, w_{s}\right)-f\left(s, \widetilde{w}_{s}\right)\right\| d s \\
& +\sum_{0<\tau_{k}<t}\left\|T\left(t-\tau_{k}\right)\right\|\left\|I_{k} w\left(\tau_{k}\right)-I_{k} \widetilde{w}\left(\tau_{k}\right)\right\| \\
\leq & M K^{*}\|w-\widetilde{w}\|_{C([-r, a], E)}+\frac{M}{\Gamma(\alpha)} \int_{0}^{t}\left\|(t-s)^{\alpha-1}\right\| \| f\left(s, w_{s}\right) \\
& -f\left(s, \widetilde{w}_{s}\right)\left\|d s+M \sum_{0<\tau_{k}<t}\right\| I_{k} w\left(\tau_{k}\right)-I_{k} \widetilde{w}\left(\tau_{k}\right) \| \\
\leq & M K^{*}\|w-\widetilde{w}\|_{C([-r, a], E)}+\frac{M}{\Gamma(\alpha)} \int_{0}^{t}\left\|(t-s)^{\alpha-1} L_{1}\right\| \| w_{s} \\
& -\widetilde{w}_{s}\left\|d s+M L_{2} \sum_{0<\tau_{k}<t}\right\| w\left(\tau_{k}\right)-\widetilde{w}\left(\tau_{k}\right) \| \\
\leq & M K^{*}\|w-\widetilde{w}\|_{C([-r, a], E)}+M t^{\alpha} L_{1}\|w-\widetilde{w}\|_{C([-r, a], E)} \\
& +M L_{2} \sum_{0<\tau_{k}<t}\left\|w\left(\tau_{k}\right)-\widetilde{w}\left(\tau_{k}\right)\right\| \\
\leq & M\left(K^{*}+t^{\alpha} L_{1}+p L_{2}\right)\|w-\widetilde{w}\|_{C([-r, a], E)} \\
\leq & \|w-\widetilde{w}\|_{C([-r, a], E)},
\end{aligned}
$$

where $t \in[0, a]$. This ends the proof that $F$ is a contraction operator on $C([-r, a], E)$. It follows that $F$ has a fixed point $F u(t)=u(t)$ and hence

$$
\begin{aligned}
& u^{\alpha}(t)=A u(t)+f\left(t, u_{t}\right), \\
& u(t)+\left(g\left(u_{t_{1}}, \ldots, u_{t_{p}}\right)\right)(t)=\phi(t), t \in[-r, 0] .
\end{aligned}
$$

Therefore, the fixed point $u(t)$ is a mild solution of the nonlocal Cauchy problem.

Theorem 2.2. Suppose that the functions $f$ and $g$ satisfy assumptions (H1)-(H3) and $M\left(K^{*}+t^{\alpha} L_{1}+p L_{2}\right)<$ 1. Then for each $\phi_{1}, \phi_{2} \in C([-r, 0], E)$ and for the corresponding mild solutions $u_{1}(t), u_{2}(t)$ of the problem

$$
\begin{aligned}
& u^{\alpha}(t)=A u(t)+f\left(t, u_{t}\right), t \in(0, a], t \neq \tau_{k}, \\
& u\left(\tau_{k}+0\right)=Q_{k} u\left(\tau_{k}\right) \equiv u\left(\tau_{k}\right)+I_{k} u\left(\tau_{k}\right), k=1,2, \ldots, K, \\
& u(t)+\left(g\left(u_{t_{1}}, \ldots, u_{t_{p}}\right)\right)(t)=\phi_{i}(t), t \in[-r, 0](i=1,2),
\end{aligned}
$$

the inequality

$$
\left\|u_{1}-u_{2}\right\|_{C([-r, t], E)} \leq C_{*}+C_{*} \sum_{n=1}^{\infty} \frac{M^{n} L_{1}^{n}}{\Gamma(n \alpha+1)} t^{n \alpha},
$$

where $C_{*}=M\left(\left\|\phi_{1}-\phi_{2}\right\|_{C([-r, 0], E)}+K^{*}\left\|u_{1}-u_{2}\right\|_{C([-r, a)], E)}+L_{2} \sum_{0<\tau_{k}<\tau}\left\|u_{1}\left(\tau_{k}\right)-u_{2}\left(\tau_{k}\right)\right\|_{C([-r, a], E)}\right)$ is hold. 
Proof. Let $\phi_{1}, \phi_{2} \in C([-r, 0], E)$ and let $u_{1}(t), u_{2}(t)$ be the mild solutions of nonlocal Cauchy problem. Then for every $t \in[-r, 0)$

$$
u_{1}(t)-u_{2}(t)=\phi_{1}(t)-\phi_{2}(t)+\left(g\left(\left(u_{2}\right)_{t_{1}}, \ldots,\left(u_{2}\right)_{t_{p}}\right)\right)(t)-\left(g\left(\left(u_{1}\right)_{t_{1}}, \ldots,\left(u_{1}\right)_{t_{p}}\right)(t)\right)
$$

and by assumption (H3) we have

$$
\left\|u_{1}(t)-u_{2}(t)\right\| \leq\left\|\phi_{1}(t)-\phi_{2}(t)\right\|_{C([-r, 0), E)}+K^{*}\left\|u_{1}(t)-u_{2}(t)\right\|_{C([-r, a], E)} .
$$

Also, for every $t \in[0, a]$

$$
\begin{aligned}
u_{1}(t)-u_{2}(t)= & T(t)\left[\phi_{1}(0)-\phi_{2}(0)\right] \\
& -T(t)\left[\left(g\left(\left(u_{1}\right)_{t_{1}}, \ldots,\left(u_{1}\right)_{t_{p}}\right)\right)(0)-\left(g\left(\left(u_{2}\right)_{t_{1}}, \ldots,\left(u_{2}\right)_{t_{p}}\right)\right)(0)\right] \\
& +\frac{1}{\Gamma(\alpha)} \int_{0}^{t}(t-s)^{\alpha-1} T(t-s)\left[f \left(s,\left(u_{1}\right)_{s}-f\left(s,\left(u_{2}\right)_{s}\right] d s\right.\right. \\
& +\sum_{0<\tau_{k}<t} T\left(t-\tau_{k}\right)\left(I_{k} u_{1}\left(\tau_{k}\right)-I_{k} u_{2}\left(\tau_{k}\right)\right) .
\end{aligned}
$$

Therefore, by assumptions (H2) and (H3) we have

$$
\begin{aligned}
\left\|u_{1}(\tau)-u_{2}(\tau)\right\| \leq & M\left\|\phi_{1}(\tau)-\phi_{2}(\tau)\right\|_{C([-r, 0], E)}+M K^{*}\left\|u_{1}(\tau)-u_{2}(\tau)\right\|_{C([-r, a)], E)} \\
& +\frac{M}{\Gamma(\alpha)} \int_{0}^{\tau}(t-s)^{\alpha-1} L_{1}\left[\left\|\left(u_{1}\right)(s)-\left(u_{2}\right)(s)\right\|_{C([-r, s], E)}\right] d s \\
& +M L_{2}\left(\sum_{0<\tau_{k}<\tau}\left\|u_{1}\left(\tau_{k}\right)-u_{2}\left(\tau_{k}\right)\right\|_{C([-r, a], E)}\right) \\
\leq & M\left\|\phi_{1}-\phi_{2}\right\|_{C([-r, 0], E)}+M K^{*}\left\|u_{1}-u_{2}\right\|_{C([-r, a)], E)} \\
& \frac{M}{\Gamma(\alpha)} \int_{0}^{t}(t-s)^{\alpha-1} L_{1}\left[\left\|u_{1}-u_{2}\right\|_{C([-r, s], E)}\right] d s \\
& +M L_{2} \sum_{0<\tau_{k}<\tau}\left\|u_{1}\left(\tau_{k}\right)-u_{2}\left(\tau_{k}\right)\right\|_{C([-r, a], E)} \\
\leq & C_{*}+\frac{M}{\Gamma(\alpha)} \int_{0}^{t}(t-s)^{\alpha-1} L_{1}\left[\left\|u_{1}-u_{2}\right\|_{C([-r, s], E)}\right] d s
\end{aligned}
$$

where $C_{*}=M\left(\left\|\phi_{1}-\phi_{2}\right\|_{C([-r, 0], E)}+K^{*}\left\|u_{1}-u_{2}\right\|_{C([-r, a)], E)}+L_{2} \sum_{0<\tau_{k}<\tau}\left\|u_{1}\left(\tau_{k}\right)-u_{2}\left(\tau_{k}\right)\right\|_{C([-r, a], E)}\right)$ for $0 \leq \tau \leq t \leq a$. Therefore,

$$
\sup _{\tau \in[0, t]}\left\|u_{1}(\tau)-u_{2}(\tau)\right\| \leq C_{*}+\frac{M}{\Gamma(\alpha)} \int_{0}^{t}(t-s)^{\alpha-1} L_{1}\left[\left\|u_{1}-u_{2}\right\|_{C([-r, s], E)}\right] d s
$$

for $t \in[0, a]$. At the same time, by (H3) and above we have

$$
\left\|u_{1}(t)-u_{2}(t)\right\| \leq M\left\|\phi_{1}(t)-\phi_{2}(t)\right\|_{C([-r, 0), E)}+M K^{*}\left\|u_{1}(t)-u_{2}(t)\right\|_{C([-r, a], E)}
$$

for $t \in[-r, 0)$. Formulas $(*)$ and $(* *)$ and generalized Gronwall inequality (see [11]) imply that

$$
\begin{aligned}
\left\|u_{1}-u_{2}\right\|_{C([-r, t], E)} & \leq C_{*}+\frac{M L_{1}}{\Gamma(\alpha)} \int_{0}^{t}(t-s)^{\alpha-1}\left\|u_{1}-u_{2}\right\|_{C([-r, s], E)} d s \\
& \leq C_{*}+\int_{0}^{t} C_{*} \sum_{n=1}^{\infty} \frac{M^{n} L_{1}^{n}}{\Gamma(n \alpha)}(t-s)^{n \alpha-1} d s \\
& \leq C_{*}+C_{*} \sum_{n=1}^{\infty} \frac{M^{n} L_{1}^{n}}{\Gamma(n \alpha+1)} t^{n \alpha} .
\end{aligned}
$$

Therefore, the inequality is hold. 


\section{References}

[1] J. H. Barrett, Differential equations of non-integer order, Canadian J. Math., 6 (1954), 529-541. 1

[2] L. Byszewski, H. Akca, On a mild solution of a semlinear functional differential evolution nonlocal problem, Int. J. Stoch. Anal., 10 (1997), 265-271. 1

[3] M. Caputao, Linear models of dissipation whose $Q$ is almost frequency independent, Geophys. J. R. Astr. Soc., 13 (1967), 529-539. 1

[4] D. Delbosco, L. Rodino, Existence and uniqueness for a nonlinear fractional differential equation, J. Math. Anal. Appl., 204 (1996), 609-625. 1

[5] J. H. He, Approximate analytical solution for seepage flow with fractional derivatives in porous media, Comput. Methods Appl. Mech. Engrg., 167 (1998), 57-68. 1

[6] J. H. He, Some applications of nonliear fractional differential equations and their approximations, Bull. Sci. Technol., 15 (1999), 86-90 . 1

[7] V. Lakshmikantham, D. D. Bainov, P. S. Simeonov, Theory of Impulsive Differential Equations, World Scientific Publishing Co., Inc., Teaneck, NJ, (1989). 1

[8] F. Mainardi, Fractional calculus: Some basic problem in continuum and statistical mechanics, Springer, Vienna, (1997). 1

[9] K. S. Miller, B. Ross, An introduction to the Fractional calculus and Fractional Differential Equations, John Wiley and Sons Inc., New York, (1993). 1

[10] A. M. Samoilenko, N. A. Perestyuk, Impulsive Differential Equations, World Scientific Publishing Co., Inc., River Edge, New Jersey, (1995). 1

[11] H. Ye, G. D. Gao, Y. Ding, A generalized Gronwall inequality and its application to a fractional differential equation, J. Math. Anal. Appl., 328 (2006), 1075-1081. 2 